\title{
Síntesis por el método sol-gel aplicado al estudio del polimorfismo en nanopartículas de $\mathrm{TiO}_{2}$
}

\author{
M. P. Gutiérrez* y M. A. Castellanos
}

RESUMEN. Este trabajo tiene como objetivo implementar una práctica de laboratorio en la asignatura de química de materiales que forma parte del nuevo programa de la carrera de química, de la Facultad de Química de la UNAM. El trabajo incluye la elaboración de un guión a seguir por los alumnos, el cual les permitirá cumplir con los objetivos de la práctica. Estos objetivos comprenden familiarizarse con la utilización del método sol-gel para sintetizar partículas nanométricas de un óxido cerámico de importancia tecnológica e industrial como el dióxido de titanio, $\mathrm{TiO}_{2}$ y realizar el estudio de la transformación estructural del $\mathrm{TiO}_{2}$, en función de la temperatura. Durante el desarrollo de la práctica el alumno aprenderá cuáles son las variables que se deben controlar en el método sol-gel y cuáles son las ventajas y desventajas del uso de este método de síntesis. Una vez obtenido el material, hará uso de la técnica de difracción de rayos $X$ de polvos para identificar el producto y para realizar el estudio del cambio de fase estructural, en sus variedades polimórficas, que sufren las nanopartículas de $\mathrm{TiO}_{2}$, en función de los tratamientos térmicos que se realizarán en un intervalo de temperaturas comprendidas entre los 500 y los $800^{\circ} \mathrm{C}$. Además, los alumnos determinarán el tamaño promedio de las nanopartículas obtenidas de los polimorfos de $\mathrm{TiO}_{2}$. PaLABRAS CLAVE: nanopartículas de $\mathrm{TiO}_{2}$, método sol-gel, polimorfismo.

ABSTRACT: The aim of this work is to design a laboratory experiment for the Materials Chemistry course which is part of the new chemistry undergraduate program in the Facultad de Química-UNAM. The included guideline will allow the students to achieve the aims of the laboratory experiment. They will become familiar with the use of the sol-gel method to synthesize nanometric ceramic particles which have important industrial and technological applications such as titanium dioxide $\left(\mathrm{TiO}_{2}\right)$. The students will learn the advantages and disadvantages of using the sol-gel method and which variables should be controlled in the experiment. Once the students have synthesized the product they will use the $x$-ray powder diffraction technique to determine the particle size of the material. The students will also investigate the structural transition of the $\mathrm{TiO}_{2}$ as a function of the temperature in the $500-800^{\circ} \mathrm{C}$ range.

KEYWORDS: $\mathrm{TiO}_{2}$ nanoparticles, sol-gel method, polymorphism.

\section{IMPORTANCIA DE LA DIVULGACIÓN}

La educación química en Química de Materiales en nuestro país es incipiente y escasa. Esto es un tema relevante debido a que constituye una subdisciplina de la Química que emerge vigorosamente apenas hace unos veinte años y aún cuando se sigue discutiendo su definición, ahora se está desplantando, de ella y de manera acelerada, la nanoquímica. La importancia de la Química de Materiales reside en la gran diversidad de técnicas de síntesis originales y diferentes, a las de la química tradicional, para obtener nuevos compuestos de materiales sólidos con propiedades de interés tecnológico. Estas propiedades son especialmente interesantes cuando se manifiestan en substancias con tamaño de partícula a nivel nanométrico. El estudio de substancias

\footnotetext{
* Facultad de Química, Universidad Nacional Autónoma de México: e-mail: mpga@unam.mx
} 
sólidas requiere de técnicas de caracterización particulares, como la difracción de rayos X, para poder identificar la estructura cristalina presente de un producto de síntesis. El polimorfismo es un fenómeno que se presenta sólo en substancias sólidas y las propiedades que se manifiestan en cada polimorfo tienen aplicaciones muy diferentes. En los últimos diez años este fenómeno ha sido muy estudiado especialmente en la industria farmacéutica, ya que muchas veces el producir determinado polimorfo, no es fácil.

Con el propósito de ilustrar un método de síntesis, clasificado como de química suave y ampliamente utilizado en Química de Materiales, se propone el método SolGel. Se eligió como un ejemplo típico de síntesis al dióxido de titanio por ser uno de los materiales nanométricos con aplicaciones reales y con el mayor volumen de ventas. Además, porque la síntesis por sol-gel es relativamente barata, realizable en el tiempo asignado para las prácticas de laboratorio, segura, permite la observación de la formación del gel en un material que ofrece la oportunidad de observar una transición de fase a baja temperatura y con la ventaja de que es posible modular su tamaño de partícula en la escala nanométrica.

\section{INTRODUCCIÓN}

El diseño de prácticas de laboratorio para la asignatura de química de materiales, en la Facultad de Química de la UNAM, se ha planteado como objetivo el aprendizaje por parte del alumno de los diversos métodos de síntesis que existen para obtener sólidos de gran importancia actual en el área de la ciencia de los materiales. Se eligió como un ejemplo típico de síntesis al dióxido de titanio por ser uno de los materiales nanométricos con aplicaciones reales y con el mayor volumen de ventas a nivel mundial. En este sentido, cabe señalar que a pesar del enorme uso del dióxido de titanio en una gran cantidad de productos que utilizamos en la vida cotidiana, en nuestro país, la producción de este material es casi nula. Baste decir que hasta 1997, en México sólo se producía titania, $\mathrm{TiO}_{2}$, en la Planta de Dupont en Altamira, Tamaulipas, con materias primas importadas de Estados Unidos, siendo México el país que mayor cantidad de titania compró a Estados Unidos (Aguilar-Franco, 2000). Es por ello que se consideró interesante implementar como una práctica de laboratorio, la obtención de este óxido.

En cada experimento se pretende que el alumno conozca los factores que deben controlarse al emplear cada uno de los métodos aprendidos, así como las ventajas y desventajas que ofrece cada uno de ellos. Esta práctica tiene como objetivo se pretende realizar la síntesis de dióxido de titanio, en sus polimorfos de anatasa y de rutilo en forma de nanopartículas. Para lograrlo se propone utilizar el método de síntesis por sol-gel.

El dióxido de titanio, $\mathrm{TiO}_{2}$, presenta tres polimorfos a presión normal: anatasa, brookita y rutilo. Estos polimorfos carecen de toxicidad y poseen propiedades químicas, ópticas y eléctricas haciéndolos muy atractivos para diversas aplicaciones que abarcan diferentes sectores de la industria y de la tecnología avanzada. Sus aplicaciones industriales a gran escala engloban la manufactura de pigmentos, recubrimientos, plásticos, papel, fibras y textiles, tintas para impresión, refractarios, barnices, cemento blanco, cosméticos, etc. Debido a su gran compatibilidad con la piel y membranas mucosas, además de su buena dispersabilidad en soluciones orgánicas e inorgánicas, se utiliza como absorbedor de luz ultravioleta en productos de protección solar, polvos cosméticos, cremas, desodorantes y dentífricos (Aguilar Franco, 2000). En par- 
ticular, el polimorfo anatasa encuentra sus aplicaciones en áreas de alta tecnología como catálisis, donde actúa como soporte óptimo en catalizadores base para el hidrotratamiento de fracciones de petróleo; en electroquímica, como fotoelectrodo en celdas electrolíticas fotovoltaicas (Berger et al, 1993); en electrónica como capacitores ultradelgados, debido a su alta constante dieléctrica; en sensores de gas y humedad (Bernasik et al, 1993; Bersani et al, 1997; Zakrzewska et al, 1997); en recubrimientos, filtros ópticos guías de onda (Bernasik et al, 1993; Schrauzer and Guth, 1997).

Debido a sus propiedades semiconductoras, el $\mathrm{TiO}_{2}$ en su fase anatasa ha sido ampliamente estudiado en su aplicación para tratamientos de aguas y aire en donde se utiliza la fotocatálisis heterogénea, ya que este material muestra su mayor fotoactividad en la región UV-Visible (Al-Ekabi, 1993; Blake, 2001). Esta propiedad también lo hace un excelente material para la fabricación de celdas solares sensibilizadas con tintes a base de complejos de rutenio (Fahlman, 2008). La eficiencia en estas dos últimas aplicaciones requiere que la fase anatasa se encuentre en forma de nanopartículas, por lo que es importante emplear un método de síntesis que nos permita obtener esta fase pura en escala nanométrica, tal es el caso del método sol-gel.

El método sol-gel está comprendido dentro de los métodos de química suave para la obtención de materiales inorgánicos avanzados de alta tecnología que incluyen películas delgadas, fibras, partículas, etc. Gracias a que el método permite una manipulación a nivel molecular de las diversas etapas de una reacción sol-gel, es posible sintetizar nuevos materiales de alta pureza controlando las características de la microestructura. El método permite además el control de las superficies y las interfaces del material durante las últimas etapas del proceso de producción, por ejemplo, creando adecuados gradientes composicionales en la superficie. En particular, es uno de los métodos que ofrecen mayores ventajas cuando se trata de producir estructuras homogéneas a escalas extremadamente finas, es decir, del orden de nanómetros. Esta técnica permite de realizar investigaciones a escala nanométrica de los procesos químicos de hidrólisis, condensación, sinéresis, deshidratación y densificación de los materiales.

De manera general, el método sol-gel comprende dos etapas principales que son: la dispersión de partículas sólidas de tamaño coloidal (1-100 nm) en una fase líquida (formación de un sol) y la gelificación del sol; aunque se utilizan también otras variantes como son: a) hidrólisis y condensación de un alcóxido, o bien, de los nitratos precursores, seguidos por un secado hipercrítico del gel, b) hidrólisis y policondensación de los alcóxidos precursores, seguidos por un envejecimiento del gel y un secado en condiciones ambientales. Esta última es la que será utilizada en nuestro caso particular.

Cabe señalar, que el método de difracción de rayos $\mathrm{X}$ de polvos que los alumnos utilizarán en esta práctica para hacer la caracterización de su producto, lo han aprendido en una asignatura precedente que es la química del estado sólido.

\section{MÉTOdo EXPERIMENTAL}

La realización de la práctica se lleva a cabo en equipos de dos personas. Los alumnos realizan los cálculos para llevar a cabo la reacción estequiométrica siguiente:

$$
\mathrm{Ti}\left[\mathrm{O}\left(\mathrm{CH}_{2}\right)_{3} \mathrm{CH}_{3}\right]_{4(\mathrm{l})}+\mathrm{CH}_{3} \mathrm{CH}_{2} \mathrm{OH}_{(\mathrm{l})}+4 \mathrm{H}_{2} \mathrm{O}_{(\mathrm{l})} \rightarrow \mathrm{TiO}_{2(\mathrm{~s})}+\mathrm{CO}_{2(\mathrm{~g})}+\mathrm{H}_{2} \mathrm{O}_{(\mathrm{g})}
$$

En este experimento se propone realizar la síntesis del $\mathrm{TiO}_{2}$ a partir de la hidrólisis-condensación de un alcóxido de titanio (butóxido de titanio o isopropóxido de 
titanio) a temperatura ambiente, usando etanol como co-disolvente. La posterior evaporación del disolvente a $70^{\circ} \mathrm{C}$ conlleva a la formación del gel. Una vez obtenido el producto se identificará por medio de difracción de rayos $\mathrm{X}$ de polvos y se someterá a diversos tratamientos térmicos entre 500 y $800^{\circ} \mathrm{C}$, siguiendo la transformación estructural, para observar el polimorfismo del material. A través de la fórmula DebyeScherrer, el alumno será capaz de determinar el tamaño promedio de las partículas, en cada uno de los productos después de cada tratamiento térmico.

\section{RESULTAdOS Y DISCUSIÓN}

La realización de esta práctica con varios grupos del laboratorio de química de materiales ha permitido establecer la obtención del dióxido de titanio en su fase de anatasa a una temperatura de $500^{\circ} \mathrm{C}$, identificado con los datos reportados en el archivo 78-2486 del JCPDS. El tamaño promedio de partículas medido a través del patrón de difracción de polvos, utilizando la ecuación de Debye-Scherrer fue de $24 \mathrm{~nm}$. Se encontró que a medida que se incrementa la temperatura del tratamiento, el $\mathrm{TiO}_{2}$ sufre una transformación polimórfica de anatasa a rutilo entre los 700 y $800^{\circ} \mathrm{C}$. A $800^{\circ} \mathrm{C}$ se encontró la formación de la fase rutilo en forma pura, identificada con el archivo JCPDS 89-0554, con un tamaño promedio de partícula, cuyo valor se encontró en $40 \mathrm{~nm}$. Como ejemplo de estos resultados se muestra un conjunto de difractogramas en la figura 1. La figura ilustra la evolución de las fases presentes en función de la temperatura.

FIGURA 1. Difractogramas de rayos $X$ de polvos del $\mathrm{TiO}_{2}$ bajo diversos tratamientos térmicos.

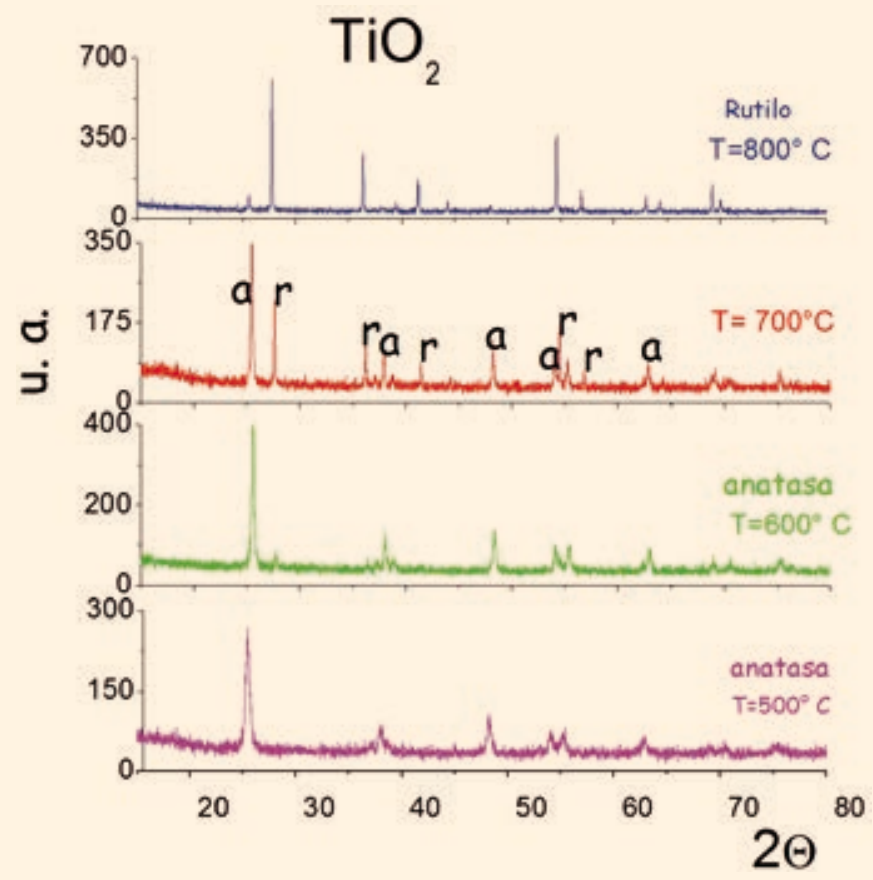

$\mathrm{a}=$ anastasa (JCPDS 78-2486).

$r=$ rutilio (JCPDS 89-0554). 
Durante el desarrollo de la práctica, los alumnos aprendieron que factores tales como velocidad de agitación, velocidad de adición de los reactivos, control de la temperatura de evaporación, etc., son importantes para la buena formación del gel. Aplicaron el método de difracción de rayos $\mathrm{X}$ de polvos para caracterizar los productos en función de la temperatura de tratamiento térmico final y para determinar el tamaño promedio de las partículas presentes. Investigaron las diversas aplicaciones que tiene el $\mathrm{TiO}_{2}$, especialmente en su fase de anatasa.

\section{Conclusión}

Se ha elaborado el guión a seguir por los alumnos, quienes ya han ensayado el desarrollo de esta práctica en equipos de dos personas, en varios grupos del laboratorio de química de materiales, obteniendo resultados reproducibles.

\section{SíNTESIS POR EL MÉTOdO SOL-GEL APLICADO AL ESTUDIO DEL POLIMORFISMO EN EL $\mathrm{TIO}_{2}^{*}$}

\section{Problema}

Demostrar la transición de fase que sufre el $\mathrm{TiO}_{2}$, en función de la temperatura, cuando se obtiene por el método de sol-gel y evaluar el tamaño promedio de partícula de los productos.

\begin{tabular}{|l|l|l|}
\hline \multicolumn{1}{|c|}{ Sustancias } & \multicolumn{1}{|c|}{ Material } & \multicolumn{1}{c|}{ Equipo } \\
\hline Butóxido de titanio & $\begin{array}{l}\text { Vasos de precipitados de } \\
250 \mathrm{~mL} \\
\text { Pipeta de } 25 \mathrm{~mL} \\
1 \text { bureta } \\
\text { Pinzas para bureta } \\
1 \text { termómetro }\left(100^{\circ} \mathrm{C}\right)\end{array}$ & $\begin{array}{l}\text { 1 balanza } \\
1 \text { Parrilla de calentamiento con agitación } \\
\text { magnética }\end{array}$ \\
\hline Etanol Absoluto & 1 mortero de ágata & Horno $\left(800{ }^{\circ} \mathrm{C}\right)$ \\
\hline Agua destilada & 2 crisoles de cerámica & \\
\hline & $\begin{array}{l}\text { Pinzas para horno } \\
1 \text { espátula }\end{array}$ & \\
\hline
\end{tabular}

Procedimiento

a. Hacer los cálculos necesarios para obtener $2 \mathrm{~g} \mathrm{de} \mathrm{TiO}_{2}$, a partir de butóxido de titanio y agua.

* Con la colaboración de Nadia Córdova Castañón. 
b. En un vaso de precipitados de $250 \mathrm{~mL}$ bien seco, coloque $15 \mathrm{~mL}$ de etanol*y agregue el butóxido de titanio, $\mathrm{Ti}\left(\mathrm{OC}_{4} \mathrm{H}_{9}\right)_{4}$. Utilice una pipeta bien seca para añadirlo.

c. Añada gota a gota el agua destilada, empleando una bureta, mientras mantiene una agitación vigorosa de la solución. Continúe agitando durante 10 minutos.

d. Caliente lentamente el sistema, cuidando que la temperatura no exceda los $70^{\circ} \mathrm{C}$ y mantenga esta temperatura y agitación lenta, hasta evaporar toda el agua residual.

e. Vacíe el producto en un crisol de cerámica y seque el producto en una estufa a $100^{\circ} \mathrm{C}$.

f. Realice un calentamiento a $500^{\circ} \mathrm{C}$, empezando desde temperatura ambiente.

g. Haga un análisis del producto por difracción de rayos $\mathrm{X}$ de polvos, determine las fases presentes y mida el tamaño promedio de las nanopartículas.

h. Muela la muestra y divídala en dos partes. Calcine una parte a $700^{\circ} \mathrm{C}$ y otra parte a $800^{\circ} \mathrm{C}$.

i. Utilice difracción de rayos X para identificar las fases presentes en cada muestra y mida el tamaño promedio de las nanopartículas en la muestra tratada a $800^{\circ} \mathrm{C}$.

* Es muy importante utilizar etanol absoluto para evitar la hidrólisis previa del butóxido.

Medidas de seguridad:

a) Usar lentes de seguridad y guantes de asbesto para el manejo del horno.

b) Lavar con etanol la pipeta usada para medir el butóxido de titanio, ¡NO CON AGUA!

\section{Cuestionario}

1. Anote la ecuación balanceada y los cálculos realizados para preparar el $\mathrm{TiO}_{2}$.

2. ¿Cuál es la fase de $\mathrm{TiO}_{2}$ obtenida después del tratamiento de $500^{\circ} \mathrm{C}$ ?

3. ¿Qué tamaño promedio de las nanopartículas presenta la fase de $500^{\circ} \mathrm{C}$ ? Describa la estructura de esta fase.

4. ¿Qué observa en el difractograma de la muestra tratada a $700^{\circ} \mathrm{C}$ ?

5. a) ¿Qué fase está presente en el producto de $800^{\circ} \mathrm{C}$ ?, b) Si regresara este producto a $500^{\circ} \mathrm{C}$, ¿qué fase(s) esperaría observar?

6. ¿Cuál es el tamaño promedio de partícula de la fase de $800^{\circ} \mathrm{C}$ ? Describa la estructura de esta fase.

7. Anote las temperaturas de transición de los principales polimorfos del $\mathrm{TiO}_{2}$.

8. ¿Cuáles son las ventajas y desventajas de haber sintetizado el $\mathrm{TiO}_{2}$ utilizando el método sol-gel, comparándolo con una síntesis por reacción en estado sólido?

9. Describa un proceso industrial que se utilice para fabricar dióxido de titanio y mencione cuál es la fase polimórfica obtenida por ese proceso.

10. Describa una aplicación específica del $\mathrm{TiO}_{2}$ en su fase anatasa y otra aplicación en la fase de rutilo. 


\section{BIBLIOGRAFIA}

Aguilar Franco, M. (2000). La titania, ( $\mathrm{TiO}_{2}$ ) su importancia y sus aplicaciones. Trabajo monográfico de actualización, tesis de licenciatura, Facultad de Química, UNAM.

Bernasik, A., Radecka, M., Rekas, M. y Sloma, M. (1993). Applied Surface Science, 65/66: 240.

Berger, H., Tang, H. y Lévy F. (1993). Journal of Crystal Growth, 130: 108.

Bersani, D., Antonioli, G., Lucci, P. y López, T. (1997). Materials Science Forum, 87: 239.

Fahlman, B. D. (2008). Materials Chemistry. The Netherlands: Springer Science + Bussines Media B.V.

Font-Alba, M. (1996). Atlas temático de mineralogía. Barcelona: Idea Books, S. A.

Hench, L.L. y West, J. K. (1990) The sol-gel process. Chem. Rev., 90: 33-72.

Lalena, J. N., Cleary, E- A., Carpenter, E.E. y Dean, N. F. (2008). Inorganic materials synthesis and fabrication. New Jersey: John Wiley and Sons.

Matos, J., Chovelon, J. M. y Ferronato C. (2009). Mundo Nano, 1(2): 37.

Schrauzer, G. and Guth, T. (1997), Journal of the American Chemical Society, 99: 7189.

Zakrsewska, K., Radecka, M. y Rekas, M. (1997). Thin Solid Films, 310: 161. 